Research article

Open Access

\title{
Synthesis and Anticancer Activity of New Thiopyrano[2,3-d]thiazoles Based on Cinnamic Acid Amides
}

\author{
Andrii LOZYNSKYI, Borys ZIMENKovsky, Roman LeSYK *
}

Department of Pharmaceutical, Organic and Bioorganic Chemistry, Danylo Halytsky Lviv National Medical University, Pekarska 69, 79010, Lviv, Ukraine.

* Corresponding author. E-mail: dr_r_lesyk@org.Iviv.net (R. Lesyk)

Sci Pharm. 2014; 82: 723-733

doi:10.3797/scipharm.1408-05

Published: $\quad$ September $15^{\text {th }} 2014$

Accepted: $\quad$ September $15^{\text {th }} 2014$

Received: $\quad$ August $5^{\text {th }} 2014$

This article is available from: http://dx.doi.org/10.3797/scipharm.1408-05

(c) Lozynskyi et al.; licensee Österreichische Apotheker-Verlagsgesellschaft m. b. H., Vienna, Austria.

This is an Open Access article distributed under the terms of the Creative Commons Attribution License (http://creativecommons.org/licenses/by/3.0/), which permits unrestricted use, distribution, and reproduction in any medium, provided the original work is properly cited.

\begin{abstract}
Novel rel-(5R,6S,7S)-2-oxo-5-phenyl-7-aryl(hetaryl)-3,7-dihydro-2H-thiopyrano $[2,3-d]$ thiazole-6-carboxylic acid amides were synthesized in a hetero-DielsAlder reaction with a series of cinnamic acid amides. The synthesized compounds were tested for their anticancer activity in vitro in the standard National Cancer Institute 60 cancer cell line assay. Promising compounds $\mathbf{3 e}$, $\mathbf{3 g}$, and $\mathbf{3 h}$ with moderate antitumor activity were identified among the synthesized series.
\end{abstract}

\section{Keywords}

hetero-Diels-Alder reaction - Cynnamic acid amides - 5-Ylideneisorhodanines • Thiopyrano[2,3-d][1,3]thiazoles • Anticancer activity

\section{Introduction}

Investigations of thiopyrano[2,3- $d$ ] thiazole derivatives, the isosteric mimics of biologically active 5-ylidene-4-thiazolidinones, led to the synthesis of compounds with anticancer, antitrypanosomal, and antimycobacterial properties which can provide an opportunity to further study and explore the pharmacological activity of these heterocyclic systems in the future [1-13]. We decided to combine in a single heterocyclic system the thiazolidinone moiety and a fragment of cinnamic acid (Sch. 1). Cinnamic acid and its derivatives exhibit antitumor, antimicrobial, antifungal action and act as histamine $\mathrm{H}_{3}$-receptor antagonists 
[14-16]. Consequently, we have synthesized thiopyrano[2,3-d]thiazoles using cinnamic acid amides as the dienophile in the reaction of hetero-Diels-Alder.

In addition, heterodiene synthesis allows the fixing of the biologically important 4-thiazolidinone fragment in a rigid fused system, preserving its biological activity. Moreover, the combination of thiazole and thiopyran in a fused heterosystem is a precondition for creating ligand-target binding and enhances the potential selectivity to biotargets. This approach suggests the critical impact of the substituent on the biological activity with particular selectivity to various cancer cell lines.

\section{Cinnamic acid amides moiety}
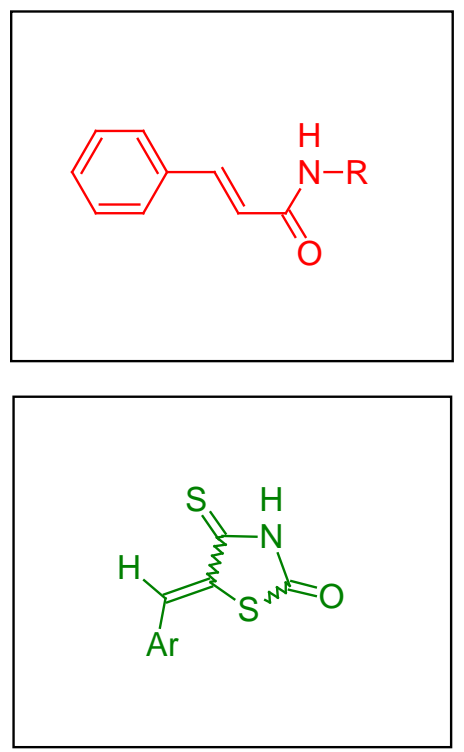

\section{Structure of target compounds}
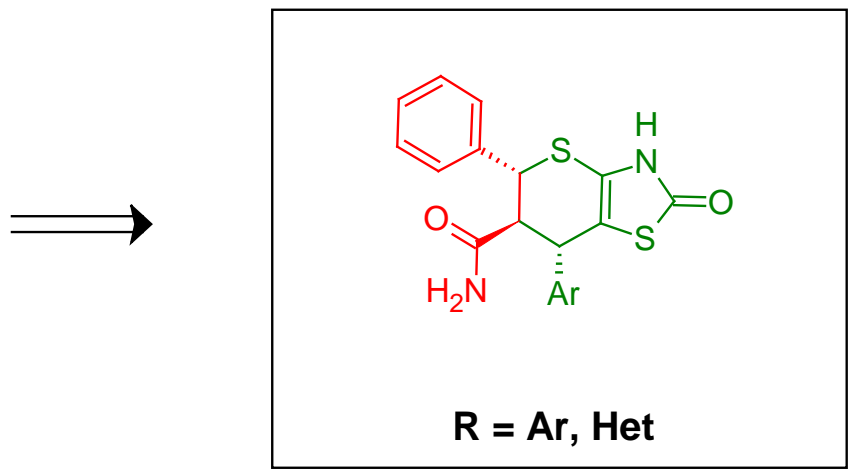

\section{5-Arylidene-4-thiazolidinone moiety}<smiles>COc1ccc([C@H]2c3sc(=O)[nH]c3SC[C@]23CC(=O)N(c2ccc(Cl)cc2)C3=O)cc1OC</smiles>

Antitrypanosomal activity

Zelisko et al., 2012 [11]<smiles>[R]c1ccccc1C1C2=C(Sc3[nH]c(=O)sc31)C(=O)c1ccccc1C2=O</smiles>

Anticancer and antimycobacterial activities

Atamanyuk et al., 2013 [10]<smiles>O=c1[nH]c2c(s1)[C@@H](c1ccc(-c3cc(Cl)ccc3Cl)o1)[C@H]1C3CCC(CC3)[C@H]1S2</smiles>

Anticancer activity

Lesyk et al., 2006 [4]

Sch. 1. Background for the synthesis of target compounds 


\section{Results and Discussion}

\section{Chemistry}

The starting 5-aryl(hetaryl)idene-4-thioxo-2-thiazolidinones $\mathbf{1 a - d}$ were obtained by the treatment of 4-thioxo-2-thiazolidinone with the appropriate aldehydes in glacial acetic acid with a catalytic amount of fused sodium acetate $[4,12]$. The cinnamic acid amides were synthesized by the interaction of the corresponding cinnamic acid chloride with 4-substituted anilines, morpholine, and 2-aminopyridine in anhydrous dioxane. The heteroDiels-Alder reaction of $\mathbf{2 a - f}$ with 5-aryl(hetaryl)idene-4-thioxo-2-thiazolidinones $\mathbf{1 a - d}$ yielded a series of novel rel-(5R,6S,7S)-2-oxo-5-phenyl-7-aryl(hetaryl)-3,7-dihydro- $2 \mathrm{H}$ thiopyrano[2,3-d]thiazole-6-carboxylic acid amides (Sch. 2).
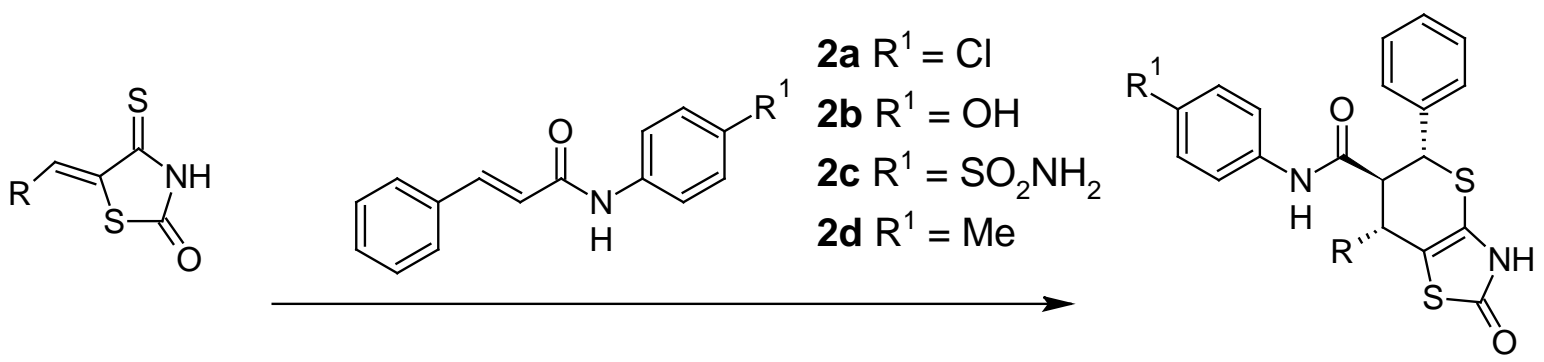

1a $\mathrm{R}=4-\mathrm{OH}-\mathrm{C}_{6} \mathrm{H}_{4}$

1b $\mathrm{R}=4-\mathrm{Cl}-\mathrm{C}_{6} \mathrm{H}_{4}$

1c $\mathrm{R}=4-\mathrm{Me}-\mathrm{C}_{6} \mathrm{H}_{4}$

1d thiophen-2-yl

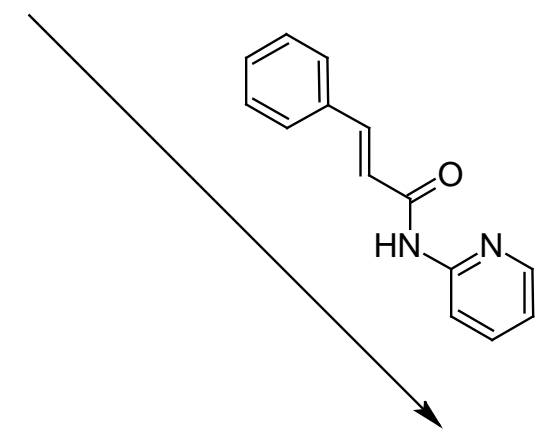

3a $\mathrm{R}=4-\mathrm{OH}-\mathrm{C}_{6} \mathrm{H}_{4}, \mathrm{R}^{1}=\mathrm{Cl}$

$\langle=0$<smiles>[R]C1c2sc(=O)[nH]c2S[C@H](c2ccccc2)C1C(=O)Nc1ccccn1</smiles>

3b R $=4-\mathrm{Cl}-\mathrm{C}_{6} \mathrm{H}_{4}, \mathrm{R}^{1}=\mathrm{OH}$

3c $\mathrm{R}=4-\mathrm{Me}-\mathrm{C}_{6} \mathrm{H}_{4}, \mathrm{R}^{1}=\mathrm{SO}_{2} \mathrm{NH}_{2}$

3d $\mathrm{R}=4-\mathrm{Cl}-\mathrm{C}_{6} \mathrm{H}_{4}, \mathrm{R}^{1}=\mathrm{SO}_{2} \mathrm{NH}_{2}$

3e $\mathrm{R}=4-\mathrm{Cl}-\mathrm{C}_{6} \mathrm{H}_{4}, \mathrm{R}^{1}=\mathrm{Cl}$

3f $\mathrm{R}=4-\mathrm{Me}-\mathrm{C}_{6} \mathrm{H}_{4}, \mathrm{R}^{1}=\mathrm{Cl}$

$3 g \mathrm{R}=4-\mathrm{Cl}-\mathrm{C}_{6} \mathrm{H}_{4}, \mathrm{R}^{1}=\mathrm{Me}$

3h $\mathrm{R}=4-\mathrm{Me}-\mathrm{C}_{6} \mathrm{H}_{4}, \mathrm{R}^{1}=\mathrm{Me}$

3i $\mathrm{R}=$ thiophen-2-yl, $\mathrm{R}^{1}=\mathrm{Cl}$

3j $\mathrm{R}=4-\mathrm{Cl}-\mathrm{C}_{6} \mathrm{H}_{4}$

$3 \mathrm{I} \quad \mathrm{R}=4-\mathrm{Cl}-\mathrm{C}_{6} \mathrm{H}_{4}$

3k $\mathrm{R}=$ thiophen-2-yl

$3 \mathrm{~m} \mathrm{R}=4-\mathrm{Me}-\mathrm{C}_{6} \mathrm{H}_{4}$

Sch. 2. Synthesis of 2-oxo-5-phenyl-7-aryl(hetaryl)-3,7-dihydro-2H-thiopyrano[2,3-d]thiazole-6-carboxylic acid amides 
The structure of the synthesized compounds was confirmed by ${ }^{1} \mathrm{H}$ - and ${ }^{13} \mathrm{C} \mathrm{NMR}$. We found the features of the stereochemistry of the above hetero-Diels-Alder reaction. Particularly, we have observed that cinnamic acid amides in the [4+2]-cyclocondensation of 5 -arylideneisorhodanines form a pair of rel-(5R,6S,7S)-diastereomers. This claim is based on the coupling constant values within $10.4-11.5 \mathrm{~Hz}$ and the spectral signals of the thiopyran fragment (triplet and two doublets at 3.40-4.87 ppm), which prove an axial-axial interaction of $5-\mathrm{H}, 6-\mathrm{H}$ and $6-\mathrm{H}, 7-\mathrm{H}$ proton pairs. Importantly, a similar pattern was observed earlier for cinnamic acids as the dienophile in the reactions of hetero-Diels-Alder $[11,12]$.

Tab. 1. Cytotoxic activity of the tested compounds in the concentration $10^{-5} \mathrm{M}$ against 60 cancer cell lines

\begin{tabular}{|c|c|c|c|}
\hline $\begin{array}{l}\text { Test } \\
\text { cpds. }\end{array}$ & $\begin{array}{l}\text { Average } \\
\text { growth, \% }\end{array}$ & $\begin{array}{l}\text { Range of } \\
\text { growth, \% }\end{array}$ & $\begin{array}{c}\text { Most sensitive cell } \\
\text { line growth, \% (cancer line/type) }\end{array}$ \\
\hline $3 b$ & 82.76 & 53.05-106.26 & $\begin{array}{l}53.05 \text { (RPMI-8226 / leukemia) } \\
61.05 \text { (SF-295 / CNS cancer) }\end{array}$ \\
\hline $3 c$ & 101.41 & $88.61-117.37$ & 88.61 (RXF 393 / renal cancer) \\
\hline $3 e$ & 57.09 & 26.38-94.10 & $\begin{array}{l}27.11 \text { (MOLT-4 / leukemia) } \\
26.38 \text { (HCT-116 / colon cancer) } \\
\text { 32.89 (SF-295 / CNS cancer) } \\
\text { 35.53 (PC-3 / prostate cancer) } \\
\text { 33.44 (MCF7 / breast cancer) } \\
33.81 \text { (T-47D / breast cancer) }\end{array}$ \\
\hline $3 g$ & 57.89 & 26.51-91.71 & $\begin{array}{l}26.51 \text { (MOLT-4 I leukemia); } \\
37.02 \text { (RPMI-8226 I leukemia) } \\
39.39 \text { (A549/ATCC I non-small cell lung cancer) } \\
32.09 \text { (HCT-116 I colon cancer) } \\
33.18 \text { (SF-295 I CNS cancer) } \\
33.99 \text { (PC-3 I prostate cancer) } \\
31.77 \text { (MCF7 I breast cancer) } \\
39.88 \text { (T-47D / breast camcer) }\end{array}$ \\
\hline $3 h$ & 77.68 & $-42.92-114.10$ & $\begin{array}{l}30.79 \text { (HOP-92 I non-small cell lung cancer) } \\
-42.92 \text { (NCI-H522 I non-small cell lung cancer) } \\
35.63 \text { (SK-MEL-5 I melanoma) } \\
-21.73 \text { (CAKI-1 I renal cancer) } \\
37.39 \text { (UO-31 I renal cancer) }\end{array}$ \\
\hline $3 i$ & 80.64 & 51.43-119.84 & $\begin{array}{l}57.75 \text { (SF-295 / CNS cancer) } \\
51.43 \text { (PC-3 / prostate cancer) } \\
59.03 \text { (MCF7 / breast cancer) }\end{array}$ \\
\hline $3 j$ & 88.76 & $61.65-112.27$ & 61.65 (SNB-75 / CNS cancer) \\
\hline $3 \mathrm{k}$ & 95.84 & $72.63-120.48$ & 72.63 ( $T-47 D /$ breast cancer) \\
\hline 31 & 100.81 & 73.58-120.80 & $\begin{array}{l}77.27 \text { (SNB-75 / CNS cancer) } \\
78.29 \text { (UO-31 / renal cancer) } \\
73.58 \text { ( } T-47 D \text { / breast cancer) }\end{array}$ \\
\hline $3 m$ & 96.07 & 73.69-110.93 & 73.69 (SR / leukemia); \\
\hline
\end{tabular}




\section{Biological Activity}

The synthesized rel-(5R,6S,7S)-2-oxo-5-phenyl-7-aryl(hetaryl)-3,7-dihydro-2H-thiopyrano[2,3- $d$ thiazole-6-carboxylic acid amides were selected by the National Cancer Institute (NCl) Developmental Therapeutic Program (www.dtp.nci.nih.gov) for the in vitro cell line screening to investigate their anticancer activity. Anticancer assays were performed according to the $\mathrm{NCl}$ protocol, which is described elsewhere [5-7, 17]. The compounds were evaluated for antitumor activity against 60 cancer lines at a $10 \mu \mathrm{M}$ concentration. The human tumor cell lines were derived from nine different cancer types: leukemia, melanoma, lung, colon, CNS, ovarian, renal, prostate, and breast cancers. The screening results are shown in Table 1.

The tested compounds showed different levels of activity on various cancer cell lines. The most active compounds were $\mathbf{3 e}, \mathbf{3 g}$, $\mathbf{3 h}$, being highly potent in certain lines of cancer, but they had almost no activity in others. Compounds $\mathbf{3 e}, \mathbf{3 g}$ have a selective effect on the growth of MOLT-4 (leukemia), HCT-116 (colon cancer), SF-295 (CNS cancer), PC-3 (prostate cancer), MCF7, and T-47D (breast cancer) cancer cell lines in comparison with others.

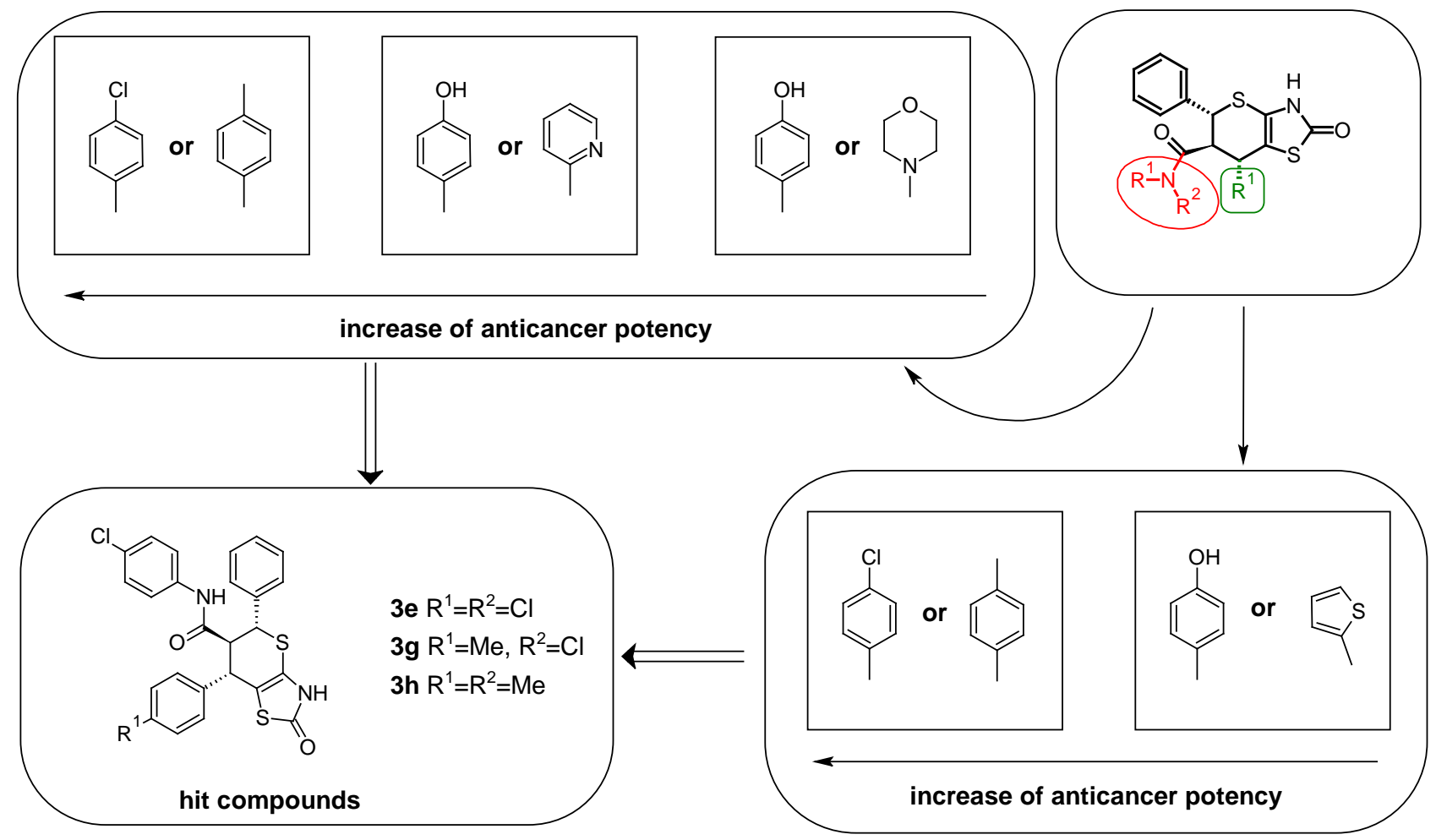

Sch. 3. SAR of anticancer potency of the synthesized thiopyrano[2,3- $d$ ] thiazole-6carboxylic acids amides

The empirical SAR study (Sch. 3) revealed that:

(1) the anticancer activity of the synthesized compounds is sensitive to the nature of the amide fragment in position 6 and substitution in position 7 of the thiopyrano[2,3-d]thiazole moiety; 
(2) introduction of $p$-Me- or $p-\mathrm{Cl}-\mathrm{C}_{6} \mathrm{H}_{4}$ groups in the amide fragment enhances the potency;

(3) the loss of anticancer activity is caused by the introduction of morpholin and pyridine fragments in position 6 or substitution of the arylamide moiety by $\mathrm{OH}$ or sulfanilamido groups;

(4) synthesized thiopyrano[2,3- $d$ ]thiazole-6-carboxylic acid amides with $p$-Me- and $p-\mathrm{Cl}-\mathrm{C}_{6} \mathrm{H}_{4}$ groups in postion 7 have the most preferable level of activity compared to other derivatives.

\section{Experimental}

\section{Chemistry}

All materials were purchased from Merck, Sigma-Aldrich, or Lancaster and were used without purification. 5-Aryl(hetaryl)idene-4-thioxo-2-thiazolidinones 1a-d were employed as starting materials and prepared according to the method described previously [4, 12]. Melting points were determined in open capillary tubes and were uncorrected. The elemental analyses ( $\mathrm{C}, \mathrm{H}, \mathrm{N})$ were performed using the Perkin-Elmer $2400 \mathrm{CHN}$ analyzer and were within $0.4 \%$ of the theoretical values. The ${ }^{1} \mathrm{H}$ - and ${ }^{13} \mathrm{C}$ NMR spectra were recorded on the Varian Gemini $400 \mathrm{MHz}$ or Bruker $125 \mathrm{MHz}$ for frequencies of $100 \mathrm{MHz}$ in DMSO- $d_{6}$ using tetramethylsilane as an internal standard. Chemical shifts are reported in ppm units with the use of a $\delta$ scale. The purity of all obtained compounds was checked by ${ }^{1} \mathrm{H}-\mathrm{NMR}$ and TLC.

\section{General Procedure of the Hetero-Diels-Alder Reaction Affording 3a-m}

A mixture of appropriate 5-aryl(hetaryl)idene-4-thioxo-2-thiazolidinone (5 mmol) and cinnamic acid amide (5.5 mmol) was refluxed for $4-7 \mathrm{~h}$ with a catalytic amount of hydroquinone (2-3 mg) in $15 \mathrm{ml}$ of glacial acetic acid and left overnight at room temperature. The obtained solid products were collected by filtration, washed with water, methanol (5-10 ml), diethyl ether, and recrystallized from the appropriate solvent.

rel-(5R,6S, 7S)-N-(4-Chlorophenyl)-7-(4-hydroxyphenyl)-2-oxo-5-phenyl-3, 5, 6, 7-tetrahydro2H-thiopyrano[2,3-d][1,3]thiazole-6-carboxamide (3a)

Yield: $59 \%, \mathrm{mp} 234-236^{\circ} \mathrm{C}(\mathrm{EtOH}) .{ }^{1} \mathrm{H}$ NMR (400 MHz, DMSO-d 6 ) $\delta: 3.47(\mathrm{t}, 1 \mathrm{H}, \mathrm{J}=10.4$ $\mathrm{Hz}, 6-\mathrm{H}), 4.21(\mathrm{~d}, 1 \mathrm{H}, \mathrm{J}=10.4 \mathrm{~Hz}, 7-\mathrm{H}), 4.83(\mathrm{~d}, 1 \mathrm{H}, \mathrm{J}=10.4 \mathrm{~Hz}, 5-\mathrm{H}), 6.70(\mathrm{~d}, 2 \mathrm{H}, \mathrm{J}=8.8$ $\mathrm{Hz}$, arom.), 7.00 (d, 2H, J = 8.8 Hz, arom.), 7.12 (d, 2H, J = 8.8 Hz, arom.), 7.20 (t, $1 \mathrm{H}, \mathrm{J}=$ $7.2 \mathrm{~Hz}$, arom.), 7.28 (t, 2H, J = 7.2 Hz, arom.), 7,46 (d, 2H, J = 7.2 Hz, arom.), 9,38 (s, 1H, $\mathrm{OH}), 10.27(\mathrm{~s}, 1 \mathrm{H}, \mathrm{NH}), 11.50(\mathrm{~s}, 1 \mathrm{H}, \mathrm{NH}) .{ }^{13} \mathrm{C}$ NMR (100 MHz, DMSO-d 6 ) $\delta: 170.7,167.3$, 156.6, 138.4, 137.3, 136.6, 130.3, 129.6, 128.5, 128.3, 128.2, 128.0, 127.1, 126.7, 121.0, 120.5, 56.3, 51.1, 42.1. Anal. Calcd for $\mathrm{C}_{25} \mathrm{H}_{19} \mathrm{ClN}_{2} \mathrm{O}_{3} \mathrm{~S}_{2}, \% \mathrm{C}, 60.66 ; \mathrm{H}, 3.87$; N, 5.66. Found, \%: C, 60.80; H, 3.80; N, 5.80.

rel-(5R,6S, 7S)-7-(4-Chlorophenyl)-N-(4-hydroxyphenyl)-2-oxo-5-phenyl-3, 5, 6,7-tetrahydro2H-thiopyrano[2,3-d][1,3]thiazole-6-carboxamide (3b)

Yield: $57 \%, \mathrm{mp} 220-224^{\circ} \mathrm{C}(\mathrm{EtOH}) .{ }^{1} \mathrm{H}$ NMR (400 MHz, DMSO-d 6 ) $\delta: 3.44(\mathrm{t}, 1 \mathrm{H}, \mathrm{J}=10.4$ $\mathrm{Hz}, 6-\mathrm{H}), 4.28(\mathrm{~d}, 1 \mathrm{H}, \mathrm{J}=10.4 \mathrm{~Hz}, 7-\mathrm{H}), 4.68(\mathrm{~d}, 1 \mathrm{H}, \mathrm{J}=10.4 \mathrm{~Hz}, 5-\mathrm{H}), 6.47$ (d, 2H, J = 8.8 
$\mathrm{Hz}$, arom.), 6.60 (d, 2H, J = 8.8 Hz, arom.), 7.00-7.40 (m, 7H, arom.), 7.50 (d, 2H, J = 7.2 $\mathrm{Hz}$, arom.), 9,20 (s, 1H, OH), $9.83(\mathrm{~s}, 1 \mathrm{H}, \mathrm{NH}), 11.53(\mathrm{~s}, 1 \mathrm{H}, \mathrm{NH}) .{ }^{13} \mathrm{C} \mathrm{NMR}(100 \mathrm{MHz}$, DMSO-d $\left.d_{6}\right) \delta: 170.6,164.5,140.2,139.4,138.8,136.3,129.2,128.9,128.6,128.3,128.2$, 128.1, 127.8, 126.9, 121.6, 114.8, 51.9, 50.8, 46.7. Anal. Calcd for $\mathrm{C}_{25} \mathrm{H}_{19} \mathrm{ClN}_{2} \mathrm{O}_{3} \mathrm{~S}_{2}, \% \mathrm{C}$, 60.66; H, 3.87; N, 5.64. Found, \%: C, 60.50; H, 3.70; N, 5.70.

rel-(5R,6S, 7S)-7-(4-Methylphenyl)-2-oxo-5-phenyl-N-(4-sulfamoylphenyl)-3,5,6,7tetrahydro-2H-thiopyrano[2,3-d][1,3]thiazole-6-carboxamide (3c)

Yield: $60 \%, \mathrm{mp} 188-190^{\circ} \mathrm{C}(\mathrm{EtOH}) .{ }^{1} \mathrm{H}$ NMR $\left(400 \mathrm{MHz}, \mathrm{DMSO}-d_{6}\right) \delta: 2.27\left(\mathrm{~s}, 3 \mathrm{H}, \mathrm{CH}_{3}\right)$, $3.95(\mathrm{t}, 1 \mathrm{H}, \mathrm{J}=11.4 \mathrm{~Hz}, 6-\mathrm{H}), 4.47(\mathrm{~d}, 1 \mathrm{H}, \mathrm{J}=11.4 \mathrm{~Hz}, 7-\mathrm{H}), 4.73(\mathrm{~d}, 1 \mathrm{H}, \mathrm{J}=11.4 \mathrm{~Hz}, 5-$ $\mathrm{H}), 6.98$ (d, 2H, J = 7.8 Hz, arom.), 7.09 (d, 2H, J = 7.8 Hz, arom.), $7.28\left(\mathrm{~s}, 2 \mathrm{H}, \mathrm{NH}_{2}\right), 7.30-$ 7.50 (m, 5H, arom.), 7.80 (d, 2H, J = 9.0 Hz, arom.), 7.87 (d, 2H, J = 9.0 Hz, arom.), 10.49 $(\mathrm{s}, 1 \mathrm{H}, \mathrm{NH}), 11.41(\mathrm{~s}, 1 \mathrm{H}, \mathrm{NH}) .{ }^{13} \mathrm{C} \mathrm{NMR}\left(100 \mathrm{MHz}, \mathrm{DMSO}-d_{6}\right) \delta: 167.6,163.8,142.0$, $140.9,138.3,136.6,134.4,129.9,129.0,127.7,126.7,121.7,120.5,118.7,118.4,104.8$, 51.1, 42.3, 42.1, 20.7. Anal. Calcd for $\mathrm{C}_{26} \mathrm{H}_{23} \mathrm{~N}_{3} \mathrm{O}_{4} \mathrm{~S}_{3}, \% \mathrm{C}, 58.08 ; \mathrm{H}, 4.31 ; \mathrm{N}, 7.82$. Found, \%: C, 58.20; H, 4.40; N, 7.80.

rel-(5R,6S, 7S)-7-(4-Chlorophenyl)-2-oxo-5-phenyl-N-(4-sulfamoylphenyl)-3,5,6,7tetrahydro-2H-thiopyrano[2,3-d][1,3]thiazole-6-carboxamide (3d)

Yield: 68\%, mp 182-184 ${ }^{\circ} \mathrm{C}(\mathrm{EtOH}) .{ }^{1} \mathrm{H}$ NMR (400 MHz, DMSO-d $\left.)_{6}\right) \delta: 3.95$ (t, $1 \mathrm{H}, \mathrm{J}=11.6$ $\mathrm{Hz}, 6-\mathrm{H}), 4.46(\mathrm{~d}, 1 \mathrm{H}, \mathrm{J}=11.6 \mathrm{~Hz}, 7-\mathrm{H}), 4.65(\mathrm{~d}, 1 \mathrm{H}, \mathrm{J}=11.6 \mathrm{~Hz}, 5-\mathrm{H}), 7.08(\mathrm{~d}, 2 \mathrm{H}, \mathrm{J}=8.8$ $\mathrm{Hz}$, arom.), 7.27 (d, 2H, J = 8.8 Hz, arom.), $7.29\left(\mathrm{~s}, 2 \mathrm{H}, \mathrm{NH}_{2}\right), 7.30-7.50(\mathrm{~m}, 5 \mathrm{H}$, arom.), $7.76(\mathrm{~d}, 2 \mathrm{H}, \mathrm{J}=8.6 \mathrm{~Hz}$, arom.), $7.83(\mathrm{~d}, 2 \mathrm{H}, \mathrm{J}=8.6 \mathrm{~Hz}$, arom.), $10.56(\mathrm{~s}, 1 \mathrm{H}, \mathrm{NH}), 11.55$ $(\mathrm{s}, 1 \mathrm{H}, \mathrm{NH}) .{ }^{13} \mathrm{C}$ NMR $\left(100 \mathrm{MHz}, \mathrm{DMSO}-d_{6}\right) \delta: 171.0,167.6,163.8,142.0,140.9,138.3$, 130.5, 129.9, 128.9, 128.4, 127.7, 126.6, 126.5, 121.7, 118.5, 104.1, 56.0, 51.0, 42.1. Anal. Calcd for $\mathrm{C}_{25} \mathrm{H}_{20} \mathrm{CIN}_{3} \mathrm{O}_{4} \mathrm{~S}_{3}, \% \mathrm{C}, 53.80 ; \mathrm{H}, 3.61 ; \mathrm{N}, 7.53$. Found, \%: $\mathrm{C}, 53.70 ; \mathrm{H}$, 3.80; N, 7.40.

rel-(5R,6S, 7S)-N,7-Bis(4-chlorophenyl)-2-oxo-5-phenyl-3,5,6, 7-tetrahydro-2Hthiopyrano[2,3-d][1,3]thiazole-6-carboxamide (3e)

Yield: $65 \%, \mathrm{mp} 216-218^{\circ} \mathrm{C}(\mathrm{EtOH}) .{ }^{1} \mathrm{H}$ NMR (400 MHz, DMSO-d 6 ) $\delta: 3.44(\mathrm{t}, 1 \mathrm{H}, \mathrm{J}=10.4$ $\mathrm{Hz}, 6-\mathrm{H}), 4.31(\mathrm{~d}, 1 \mathrm{H}, \mathrm{J}=10.4 \mathrm{~Hz}, 7-\mathrm{H}), 4.72(\mathrm{~d}, \mathrm{~J}=10.4 \mathrm{~Hz}, 5-\mathrm{H}), 6.96(\mathrm{~d}, 2 \mathrm{H}, \mathrm{J}=8.4 \mathrm{~Hz}$, arom.), 7.03 (d, 2H, J = 8.4 Hz, arom.), 7.16-7.31 (m, 7H, arom.), 7.44 (d, 2H, J = 7.0 Hz, arom.), $9.46(\mathrm{~s}, 1 \mathrm{H}, \mathrm{NH}), 11.31(\mathrm{~s}, 1 \mathrm{H}, \mathrm{NH}) .{ }^{13} \mathrm{C}$ NMR $\left(100 \mathrm{MHz}, \mathrm{DMSO}-d_{6}\right) \delta: 170.4$, 168.3, 139.2, 136.4, 136.1, 132.1, 131.1, 130.1, 128.5, 128.4, 128.3, 128.2, 127.3, 121.0, 120.4, 107.4, 56.1, 48.4, 44.9. Anal. Calcd for $\mathrm{C}_{25} \mathrm{H}_{18} \mathrm{Cl}_{2} \mathrm{~N}_{2} \mathrm{O}_{2} \mathrm{~S}_{2}, \% \mathrm{C}, 58.48 ; \mathrm{H}, 3.53 ; \mathrm{N}$, 5.46. Found, \%: C, 58.30; H, 3.40; N, 5.50.

rel-(5R,6S, 7S)-N-(4-Chlorophenyl)-7-(4-methylphenyl)-2-oxo-5-phenyl-3,5,6, 7-tetrahydro2H-thiopyrano[2,3-d][1,3]thiazole-6-carboxamide (3f)

Yield: $75 \%, \mathrm{mp} 200-202^{\circ} \mathrm{C}(\mathrm{EtOH}) .{ }^{1} \mathrm{H}$ NMR (400 MHz, DMSO-d $\left.{ }_{6}\right) \delta: 2.28\left(\mathrm{~s}, 3 \mathrm{H}, \mathrm{CH}_{3}\right)$, $3.44(\mathrm{t}, 1 \mathrm{H}, \mathrm{J}=10.4 \mathrm{~Hz}, 6-\mathrm{H}), 4.26(\mathrm{~d}, 1 \mathrm{H}, \mathrm{J}=10.4 \mathrm{~Hz}, 7-\mathrm{H}), 4.70(\mathrm{~d}, 1 \mathrm{H}, \mathrm{J}=10.4 \mathrm{~Hz}, 5-$ $\mathrm{H}), 6.96(\mathrm{~d}, 2 \mathrm{H}, \mathrm{J}=8.8 \mathrm{~Hz}$, arom.), $7.02(\mathrm{~d}, 2 \mathrm{H}, \mathrm{J}=8.8 \mathrm{~Hz}$, arom.), 7.05 (d, $2 \mathrm{H}, \mathrm{J}=7.6 \mathrm{~Hz}$, arom.), 7.13 (d, $2 \mathrm{H}, \mathrm{J}=7.6 \mathrm{~Hz}$, arom.), 7.18 (t, $1 \mathrm{H}, \mathrm{J}=7.2 \mathrm{~Hz}$, arom.), 7.24 (t, $2 \mathrm{H}, \mathrm{J}=7.2$ $\mathrm{Hz}$, arom.), 7.44 (d, $2 \mathrm{H}, \mathrm{J}=7.2 \mathrm{~Hz}$, arom.), $9.41(\mathrm{~s}, 1 \mathrm{H}, \mathrm{NH}), 11.23(\mathrm{~s}, 1 \mathrm{H}, \mathrm{NH}) .{ }^{13} \mathrm{C} N M R$ $\left(100 \mathrm{MHz}, \mathrm{DMSO}-d_{6}\right)$ $\delta: 170.5,168.5,137.2,136.7,136.5,136.2,129.0,128.5,128.3$, 
128.2, 128.1, 127.1, 119.8, 108.3, 56.2, 48.6, 45.2, 20.7. Anal. Calcd for $\mathrm{C}_{26} \mathrm{H}_{21} \mathrm{ClN}_{2} \mathrm{O}_{2} \mathrm{~S}_{2}$, \% C, 63.34; H, 4.29; N, 5.68. Found, \%: C, 63.50; H, 4.40; N, 5.70.

rel-(5R,6S, 7S)-7-(4-Chlorophenyl)-N-(4-methylphenyl)-2-oxo-5-phenyl-3,5,6, 7-tetrahydro$2 \mathrm{H}$-thiopyrano[2,3-d][1,3]thiazole-6-carboxamide (3g)

Yield: 70\%, mp 234-236 ${ }^{\circ} \mathrm{C}(\mathrm{EtOH}) .{ }^{1} \mathrm{H}$ NMR (400 MHz, DMSO-d 6 ) $\delta: 2.16\left(\mathrm{~s}, 3 \mathrm{H}, \mathrm{CH}_{3}\right)$, $3.44(\mathrm{t}, 1 \mathrm{H}, \mathrm{J}=10.4 \mathrm{~Hz}, 6-\mathrm{H}), 4.30(\mathrm{~d}, 1 \mathrm{H}, \mathrm{J}=10.4 \mathrm{~Hz}, 7-\mathrm{H}), 4.70(\mathrm{~d}, 1 \mathrm{H}, \mathrm{J}=10.4 \mathrm{~Hz}, 5-$ $\mathrm{H}), 6.75(\mathrm{~d}, 2 \mathrm{H}, \mathrm{J}=8.4 \mathrm{~Hz}$, arom.), $6.83(\mathrm{~d}, 2 \mathrm{H}, \mathrm{J}=8.4 \mathrm{~Hz}, \operatorname{arom}),. 7.20-7.30(\mathrm{~m}, 7 \mathrm{H}$, arom.), 7.46 (d, $2 \mathrm{H}, \mathrm{J}=7.2 \mathrm{~Hz}$, arom.), $9.23(\mathrm{~s}, 1 \mathrm{H}, \mathrm{NH}), 11.29(\mathrm{~s}, 1 \mathrm{H}, \mathrm{NH}) .{ }^{13} \mathrm{C} N M R(100$ MHz, DMSO-d $\left.d_{6}\right) \delta: 170.4,167.9,139.3,136.2,134.9,132.7,132.0,130.2,128.6,128.5$, $128.4,128.3,120.4,119.8,119.1,107.5,55.8,48.5,45.0,20.3$. Anal. Calcd for $\mathrm{C}_{26} \mathrm{H}_{21} \mathrm{ClN}_{2} \mathrm{O}_{2} \mathrm{~S}_{2}, \%$ C, 63.34; H, 4.29; N, 5.68. Found, \%: C, 63.20; H, 4.40; N, 5.70.

rel-(5R,6S, 7S)-N,7-Bis(4-methylphenyl)-2-oxo-5-phenyl-3,5,6,7-tetrahydro-2H-

thiopyrano[2,3-d][1,3]thiazole-6-carboxamide (3h)

Yield: $56 \%, \mathrm{mp} 230-232^{\circ} \mathrm{C}(\mathrm{EtOH}) .{ }^{1} \mathrm{H}$ NMR (400 MHz, DMSO-d $)$ ס: $2.16\left(\mathrm{~s}, 3 \mathrm{H}, \mathrm{CH}_{3}\right)$, $2.28\left(\mathrm{~s}, 3 \mathrm{H}, \mathrm{CH}_{3}\right), 3.45(\mathrm{t}, 1 \mathrm{H}, \mathrm{J}=10.4 \mathrm{~Hz}, 6-\mathrm{H}), 4.26(\mathrm{~d}, 1 \mathrm{H}, \mathrm{J}=10.4 \mathrm{~Hz}, 7-\mathrm{H}), 4.70(\mathrm{~d}$, $1 \mathrm{H}, \mathrm{J}=10.4 \mathrm{~Hz}, 5-\mathrm{H}), 6.77$ (d, 2H, J = 8.0 Hz, arom.), 6.82 (d, 2H, J=8.0 Hz, arom.), 7.06 (d, $2 \mathrm{H}, \mathrm{J}=7.6 \mathrm{~Hz}$, arom.), 7.14 (d, 2H, J = 7.6 Hz, arom.), 7.21 (t, $1 \mathrm{H}, \mathrm{J}=7.2 \mathrm{~Hz}$, arom.), 7.25 (t, $2 \mathrm{H}, \mathrm{J}=7.2 \mathrm{~Hz}$, arom.), 7.46 (d, 2H, J = 7.2 Hz, arom.), 9.18 (s, 1H, NH), 11,22 (s, $1 \mathrm{H}, \mathrm{NH}) .{ }^{13} \mathrm{C}$ NMR $\left(100 \mathrm{MHz}, \mathrm{DMSO}-d_{6}\right) \delta: 170.5,168.1,141.2,140.1,137.4,136.6$, $136.4,135.1,132.5,128.9,128.6,128.2,128.1,119.8,119.7,108.5,55.9,48.7,45.2$, 20.7, 20.3. Anal. Calcd for $\mathrm{C}_{27} \mathrm{H}_{24} \mathrm{~N}_{2} \mathrm{O}_{2} \mathrm{~S}_{2}, \% \mathrm{C}, 68.62 ; \mathrm{H}, 5.12 ; \mathrm{N}, 5.93$. Found, \%: C, $68.70 ; \mathrm{H}, 5.20 ; \mathrm{N}, 6.00$.

rel-(5R,6S, 7S)-N-(4-Chlorophenyl)-2-oxo-5-phenyl-7-(thiophen-2-yl)-3,5, 6, 7-tetrahydro-2Hthiopyrano[2,3-d][1,3]thiazole-6-carboxamide (3i)

Yield: $84 \%, \mathrm{mp} 208-210^{\circ} \mathrm{C}(\mathrm{EtOH}) .{ }^{1} \mathrm{H}$ NMR (400 MHz, DMSO-d $\left.{ }_{6}\right) \delta: 3.55(\mathrm{t}, 1 \mathrm{H}, \mathrm{J}=10.5$ $\mathrm{Hz}, 6-\mathrm{H}), 4.72(\mathrm{~d}, 1 \mathrm{H}, \mathrm{J}=10.5 \mathrm{~Hz}, 7-\mathrm{H}), 4.87(\mathrm{~d}, \mathrm{~J}=10.5 \mathrm{~Hz}, 5-\mathrm{H}), 6.92$ (dd, $1 \mathrm{H}, \mathrm{J}=5.1$, 3.6 Hz, thiophen.), 6.98 (d, $1 \mathrm{H}, \mathrm{J}=2.4 \mathrm{~Hz}$, thiophen.), 7.05 (d, $2 \mathrm{H}, \mathrm{J}=9.0 \mathrm{~Hz}$, arom.), 7.13 (d, $2 \mathrm{H}, \mathrm{J}=7.6 \mathrm{~Hz}$, arom.), 7.17 (d, $2 \mathrm{H}, \mathrm{J}=8.4 \mathrm{~Hz}$, arom.), 7.26 (t, $1 \mathrm{H}, \mathrm{J}=7.0 \mathrm{~Hz}$, arom.), 7.30 (t, $2 \mathrm{H}, \mathrm{J}=7.5 \mathrm{~Hz}$, arom.), $7.45(\mathrm{~d}, 1 \mathrm{H}, \mathrm{J}=5.1 \mathrm{~Hz}$, thiophen.), 7.51 (d, 2H, J = 7.2 Hz,

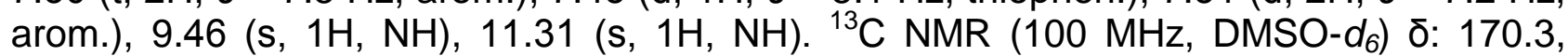
168.3, 142.8, 136.5, 136.0, 128.5, 128.4, 128.3, 128.2, 127.2, 126.7, 126.6, 125.8, 121.0, 119.9, 107.8, 59.6, 56.8, 48.6. Anal. Calcd for $\mathrm{C}_{24} \mathrm{H}_{24} \mathrm{~N}_{2} \mathrm{O}_{3} \mathrm{~S}_{2}, \%$ C, 56.95; H, 3.53; N, 5.78. Found, \%: C, 56.80; H, 3.70; N, 5.60.

rel-(5R,6S, 7S)-7-(4-Chlorophenyl)-2-oxo-5-phenyl-N-(pyridin-2-yl)-3,5, 6, 7-tetrahydro-2Hthiopyrano[2,3-d][1,3]thiazole-6-carboxamide (3j)

Yield: $56 \%, \mathrm{mp} 178-180^{\circ} \mathrm{C}(\mathrm{AcOH}) .{ }^{1} \mathrm{H}$ NMR (400 MHz, DMSO-d $\left.)_{6}\right) \delta: 3.48(\mathrm{t}, 1 \mathrm{H}, \mathrm{J}=10.5$ $\mathrm{Hz}, 6-\mathrm{H}), 4.24(\mathrm{~d}, 1 \mathrm{H}, \mathrm{J}=10.5 \mathrm{~Hz}, 7-\mathrm{H}), 4.84(\mathrm{~d}, \mathrm{~J}=10.5 \mathrm{~Hz}, 5-\mathrm{H}), 7.16-7.45(\mathrm{~m}, 9 \mathrm{H}$, arom., pyrid.), 10.21 (s, $1 \mathrm{H}, \mathrm{NH}), 11.50(\mathrm{~s}, 1 \mathrm{H}, \mathrm{NH}) .{ }^{13} \mathrm{C}$ NMR (100 MHz, DMSO-d $\left.)\right) \delta$ : $171.7,169.5,150.6,147.5,139.2,137.8,136.1,132.1,130.3,128.7,128.5,128.4,128.3$, 120.3, 119.4, 113.0, 107.5, 54.4, 48.6, 45.4. Anal. Calcd for $\mathrm{C}_{24} \mathrm{H}_{18} \mathrm{ClN}_{3} \mathrm{O}_{2} \mathrm{~S}_{2}, \% \mathrm{C}, 60.05$; H, 3.78; N, 8.75. Found, \%: C, 60.10; H, 3.70; N, 8.90. 
rel-(5R,6S, 7S)-2-Oxo-5-phenyl-N-(pyridin-2-yl)-7-(thiophen-2-yl)-3, 5, 6, 7-tetrahydro-2Hthiopyrano[2,3-d][1,3]thiazole-6-carboxamide (3k)

Yield: $76 \%, \mathrm{mp} 150-152^{\circ} \mathrm{C}(\mathrm{AcOH}) .{ }^{1} \mathrm{H}$ NMR (400 MHz, DMSO-d $\left.)_{6}\right) \delta: 3.43(\mathrm{t}, 1 \mathrm{H}, \mathrm{J}=10.5$ $\mathrm{Hz}, 6-\mathrm{H}), 4.62(\mathrm{~d}, 1 \mathrm{H}, \mathrm{J}=10.5 \mathrm{~Hz}, 7-\mathrm{H}), 4.84(\mathrm{~d}, \mathrm{~J}=10.5 \mathrm{~Hz}, 5-\mathrm{H}), 7.20-7.61(\mathrm{~m}, 9 \mathrm{H}$, arom., thiophen., pyrid.), $7.86(\mathrm{~d}, 1 \mathrm{H}, \mathrm{J}=4.0 \mathrm{~Hz}$, thiophen.), 8.10-8.20 (m, 2H, pyrid.), $10.29(\mathrm{~s}, 1 \mathrm{H}, \mathrm{NH}), 11.47(\mathrm{~s}, 1 \mathrm{H}, \mathrm{NH}) .{ }^{13} \mathrm{C}$ NMR $\left(100 \mathrm{MHz}, \mathrm{DMSO}-d_{6}\right) \delta: 171.8,170.4$, $150.8,147.5,142.5,137.7,136.1,134.6,129.3,128.7,128.5,128.3,126.6,125.9,119.8$, 113.1, 107.9, 56.1, 48.8, 47.9. Anal. Calcd for $\mathrm{C}_{22} \mathrm{H}_{17} \mathrm{~N}_{3} \mathrm{O}_{2} \mathrm{~S}_{3}, \% \mathrm{C}, 58.51 ; \mathrm{H}, 3.79 ; \mathrm{N}, 9.30$. Found, \%: C, 58.40; H, 3.90; N, 9.20.

rel-(5R,6S, 7S)-7-(4-Chlorophenyl)-6-(morpholin-4-ylcarbonyl)-5-phenyl-3,5,6,7-tetrahydro2H-thiopyrano[2,3-d][1,3]thiazol-2-one (3I)

Yield: $90 \%, \mathrm{mp} 206-208^{\circ} \mathrm{C}(\mathrm{EtOH}) .{ }^{1} \mathrm{H}$ NMR (400 MHz, DMSO-d 6 ) $\delta: 3.44(\mathrm{t}, 1 \mathrm{H}, \mathrm{J}=10.4$ $\mathrm{Hz}, 6-\mathrm{H}), 3.45-3.55(\mathrm{~m}, 4 \mathrm{H}$, morpholin), 3.73-3.81 (m, 2H, morpholin), $4.24(\mathrm{~d}, 1 \mathrm{H}, \mathrm{J}=$ $10.4 \mathrm{~Hz}, 7-\mathrm{H}), 4.64$ (d, 1H, J = $10.4 \mathrm{~Hz}, 5-\mathrm{H}), 7.10$ (d, 2H, J = 8.0 Hz, arom.), 7.18-7.34 (m, 7H, arom.), $11.33(\mathrm{~s}, 1 \mathrm{H}, \mathrm{NH}) .{ }^{13} \mathrm{C}$ NMR (100 MHz, DMSO-d 6 ) $\delta: 170.7,166.8,138.8$, $131.8,131.0,128.2,128.0,129.9,127.9,127.8,120.9,104.6,66.5,66.1,56.0,45.3,42.9$, 41.4. Anal. Calcd for $\mathrm{C}_{23} \mathrm{H}_{21} \mathrm{ClN}_{2} \mathrm{O}_{3} \mathrm{~S}_{2}, \%$ C, 58.40; $\mathrm{H}, 4.47 ; \mathrm{N}, 5.92$. Found, \%: C, 58.50; $\mathrm{H}, 4.30 ; \mathrm{N}, 6.00$.

rel-(5R,6S, 7S)-7-(4-Methylphenyl)-6-(morpholin-4-ylcarbonyl)-5-phenyl-3,5,6,7-tetrahydro2H-thiopyrano[2,3-d][1,3]thiazol-2-one (3m)

Yield: $77 \%, \mathrm{mp} 176-178^{\circ} \mathrm{C}(\mathrm{PhH}) .{ }^{1} \mathrm{H}$ NMR (400 MHz, DMSO-d 6 ) $\delta: 2.33\left(\mathrm{~s}, 3 \mathrm{H}, \mathrm{CH}_{3}\right)$, 2.77-2.86 (m, 4H, morpholin), 2.92-2.95 (m, 2H, morpholin), $3.76(\mathrm{~T}, 1 \mathrm{H}, \mathrm{J}=10.4 \mathrm{~Hz}, 6-$ $\mathrm{H}), 4.14(\mathrm{~d}, 1 \mathrm{H}, \mathrm{J}=10.4 \mathrm{~Hz}, 7-\mathrm{H}), 4.69(\mathrm{~d}, 1 \mathrm{H}, \mathrm{J}=10.4 \mathrm{~Hz}, 5-\mathrm{H}), 7.10$ (br.s, 4H, arom.), 7.25-7.34 (m, 3H, arom.), 7.40 (d, $2 \mathrm{H}, \mathrm{J}=7.0 \mathrm{~Hz}$, arom.), 11.25 (s, $1 \mathrm{H}, \mathrm{NH}) .{ }^{13} \mathrm{C} N M R$ $\left(100 \mathrm{MHz}, \mathrm{DMSO}-d_{6}\right) \delta: 170.5,168.7,146.3,145.3,136.9,136.8,128.9,128.5,128.3$, 128.2, 120.1, 65.6, 49.2, 48.7, 45.7, 45.3, 41.3, 20.9. Anal. Calcd for $\mathrm{C}_{24} \mathrm{H}_{24} \mathrm{~N}_{2} \mathrm{O}_{3} \mathrm{~S}_{2}, \%$ C, 58.40; H, 4.47; N, 5.92. Found, \%: C, 58.30; H, 4.50; N, 5.80.

\section{Cytotoxic Activity Against Malignant Human Tumor Cells}

An anticancer in vitro assay was performed on the human tumor cell lines panel derived from nine neoplastic diseases, in accordance with the protocol of the Drug Evaluation Branch, National Cancer Institute, Bethesda [5-7, 17]. The tested compounds were added to the culture at a single concentration $\left(10^{-5} \mathrm{M}\right)$ and the cultures were incubated for $48 \mathrm{~h}$. Endpoint determinations were made with a protein binding dye, sulforhodamine B (SRB). Results for each tested compound were reported as the percent of growth of the treated cells when compared to the untreated control cells. The growth percentage was evaluated spectrophotometrically versus controls not treated with the test agents.

\section{Acknowledgement}

We thank Dr. V.L. Narayanan from the Drug Synthesis and Chemistry Branch, National Cancer Institute, Bethesda, MD, USA, for the in vitro evaluation of anticancer activity. The authors support all people of good will currently struggling for sovereign and unified Ukraine. 


\section{Authors' Statement}

\section{Competing Interests}

The authors declare no conflict of interest.

\section{References}

[1] Lesyk RB, Zimenkovsky BS.

4-Thiazolidones: Centenarian History, Current Status and Perspectives for Modern Organic and Medicinal Chemistry.

Curr Org Chem. 2004; 8: 1547-1577.

http://dx.doi.org/10.2174/1385272043369773

[2] Lesyk RB, Zimenkovsky BS, Kaminskyy DV, Kryshchyshyn AP, Havryluk DYa, Atamanyuk DV, Subtel'na IYu, Khyluk DV.

Thiazolidinone motif in anticancer drug discovery. Experience of DH LNMU medicinal chemistry scientific group.

Biopolym Cell. 2011; 27: 107-117.

http://dx.doi.org/10.7124/bc.000089

[3] Kaminskyy D, Vasylenko O, Atamanyuk D, Gzella A, Lesyk R.

Isorhodanine and Thiorhodanine Motifs in the Synthesis of Fused Thiopyrano[2,3-d][1,3]thiazoles.

Synlett. 2011; 1385-1388.

http://dx.doi.org/10.1055/s-0030-1260765

[4] Lesyk R, Zimenkovsky B, Atamanyuk D, Jensen F, Kiec-Kononowicz K, Gzella A.

Anticancer thiopyrano[2,3-d][1,3]thiazol-2-ones with norbornane moiety. Synthesis, cytotoxicity, physico-chemical properties, and computational studies.

Bioorg Med Chem. 2006; 14: 5230-5240.

http://dx.doi.org/10.1016/j.bmc.2006.03.053

[5] Boyd MR, Paull KD.

Some practical considerations and applications of the national cancer institute in vitro anticancer drug discovery screen.

Drug Dev Res. 1995; 34: 91-109.

http://dx.doi.org/10.1002/ddr.430340203

[6] Boyd MR.

In: Cancer Drug Discovery and Development.

Teicher BA, ed.

Volume 2, Totowa, NJ, USA: Humana Press, 1997: 23-43.

[7] Shoemaker $\mathrm{RH}$.

The $\mathrm{NCl} 60$ human tumour cell line anticancer drug screen.

Nat Rev Cancer. 2006; 6: 813-823.

http://dx.doi.org/10.1038/nrc1951

[8] Atamanyuk D, Zimenkovsky B, Lesyk R.

Synthesis and anticancer activity of novel thiopyrano[2.3-d]thiazole-based compounds containing norbornane moiety.

J Sulfur Chem. 2008; 29: 151-162.

http://dx.doi.org/10.1080/17415990801911723

[9] Kryshchyshyn A, Atamanyuk D, Lesyk R.

Fused Thiopyrano[2,3-d]thiazole Derivatives as Potential Anticancer Agents

Sci Pharm. 2012; 80: 509-529.

http://dx.doi.org/10.3797/scipharm.1204-02 
[10] Atamanyuk D, Zimenkovsky B, Atamanyuk V, Nektegayev I, Lesyk R.

Synthesis and Biological Activity of New Thiopyrano[2,3-d][1,3]thiazoles Containing a Naphthoquinone Moiety.

Sci Pharm. 2013; 81: 423-436.

http://dx.doi.org/10.3797/scipharm.1301-13

[11] Zelisko N, Atamanyuk D, Vasylenko O, Grellier P, Lesyk R.

Synthesis and antitrypanosomal activity of new 6,7,7-trisubstituted thiopyrano[2,3-d][1,3]thiazoles.

Bioorg Med Chem Lett. 2012; 22: 7071-7074.

http://dx.doi.org/10.1016/j.bmcl.2012.09.091

[12] Zelisko N, Atamanyuk D, Vasylenko O, Bryhas A, Matiychuk V, Gzella A, Lesyk R.

Crotonic, cynnamic and propiolic acids motifs in the synthesis of thiopyrano[2,3-d][1,3]thiazoles via hetero-Diels-Alder reaction and related tandem processes.

Tetrahedron. 2013; 70: 720-729.

http://dx.doi.org/10.1016/j.tet.2013.11.083

[13] Tomasic T, Masic LP.

Rhodanine as a scaffold in drug discovery: a critical review of its biological activities and mechanisms of target modulation.

Expert Opin Drug Discov. 2012; 7: 549-560.

http://dx.doi.org/10.1517/17460441.2012.688743

[14] Lau J, Jeppsen C, Rimvall K, Hohlweg R.

Ureas with histamine $\mathrm{H}_{3}$-antagonist receptor activity- A new scaffold discovered by lead-hopping from cinnamic acid amides.

Bioorg Med Chem Lett. 2006; 16: 5303-5308.

http://dx.doi.org/10.1016/j.bmcl.2006.07.093

[15] Shukla Y, Srivastava A, Kumar S, Kumar S.

Phytotoxic and antimicrobial constituents of Argyreia speciosa and Oenothera biennis.

J Ethnopharmacol. 1999; 67: 241-245.

http://dx.doi.org/10.1016/S0378-8741(99)00017-3

[16] Narasimhan B, Belsare D, Pharande D, Mourya V, Dhake A.

Esters, amides and substituted derivatives of cinnamic acid: synthesis, antimicrobial activity and QSAR investigations.

Eur J Med Chem. 2004; 39: 827-834.

http://dx.doi.org/10.1016/j.ejmech.2004.06.013

[17] Monks A, Scudiero D, Skehan P, Shoemaker R, Paull K, Vistica D, Hose C, Langley J, Cronise P, Vaigro-Wolff A, Gray-Goodrich M, Campbell H, Mayo J, Boyd M.

Feasibility of a High-Flux Anticancer Drug Screen Using a Diverse Panel of Cultured Human Tumor Cell Lines.

J Nat Cancer Inst. 1991; 83: 757-766.

http://dx.doi.org/10.1093/jnci/83.11.757 